\title{
Gene expression profiling of HL-60 cells following knockdown of nucleostemin using DNA microarrays
}

\author{
XIAOLI SUN ${ }^{1-4}$, YU JIA $^{1}$, YUANYU WEI ${ }^{1}$, SHUAI LIU ${ }^{1}$ and BAOHONG YUE ${ }^{1,3,4}$ \\ ${ }^{1}$ Department of Clinical Laboratory, The First Affiliated Hospital of Zhengzhou University, Zhengzhou; ${ }^{2}$ Department of Clinical \\ Laboratory, The First Affiliated Hospital of Henan University of TCM, Zhengzhou, Henan; ${ }^{3}$ Faculty of Laboratorial Medicine, \\ Zhengzhou University, Zhengzhou; ${ }^{4}$ Key-Discipline Laboratory Clinical Medicine Henan, Zhengzhou, P.R. China
}

Received February 18, 2014; Accepted April 29, 2014

DOI: $10.3892 /$ or.2014.3240

\begin{abstract}
Nucleostemin (NS) plays an important role in tumorigenesis and progression. Most studies consider that NS plays its role through combining with p53 and inhibiting it, however our previous studies revealed that NS could also function without the existence of p53. To date, few studies have focused on the p53-independent pathway of NS, and its molecular mechanism remains unknown. The aim of the present study was to investigate the $\mathrm{p} 53$-independent pathway of NS in the human acute myeloid leukemia cell line HL-60 which was p53-null by using the DNA microarray technique. Lentivirus-mediated RNA interference technique was used to knock down NS expression in HL-60 cells, and then DNA microarray and bioinformatics were used to analyze the gene expression profiling changes. The microarray data showed that after knocking down NS in HL-60 cells, 2,628 differentially expressed genes were identified through $\geq 2$ or $\leq 0.5$-foldchange, in which 818 genes were upregulated and 1,810 genes were downregulated. Real-time quantitative polymerase chain reaction (qPCR) validated the reliability of DNA microarray data. Pathway analysis showed extensive signal pathways in HL-60 cells were influenced by inhibiting NS expression. In particular, the inhibition of PI3K-AKT pathway, JAK-STAT pathway, RAS-RAF-MEK-ERK1/2 pathway and activation of JNK pathway, p38 MAPK pathway may associate with the apoptosis of HL-60 cells after knocking down NS. The findings of this study provide insight to further explore the specific molecular mechanism of NS function in p53-null leukemia and they also lay the foundations for exploring new therapeutic targets for p53-null leukemia and even p53-null tumors.
\end{abstract}

\section{Introduction}

Nucleostemin (NS) is a protein related to cell proliferation which was originally identified in 2002, and located in the

Correspondence to: Professor Baohong Yue, Department of Clinical Laboratory, The First Affiliated Hospital of Zhengzhou University, 1 Jianshe Road, Zhengzhou, Henan 450052, P.R. China E-mail: ybh2002@163.com

Key words: nucleostemin, microarray, gene expression profiling, HL-60, apoptosis nucleolus; it was first found in some early pluripotent cells such as embryonic, neural and bone marrow-derived stem cells in rat, but was not expressed in terminally differentiated cells $(1,2)$. NS is highly expressed in several types of tumor tissues and cells, such as prostate and esophageal cancer $(3,4)$. After knocking down NS expression in tumor cells, its proliferation became weak, and most studies suggest that NS regulates cell proliferation through blocking the cells at the $\mathrm{G} 0$ / G1 or G2/M point and then leads the cells out of the cell cycle (5-7). These studies indicated that NS is essential to maintain proliferation and undifferentiated state of stem and tumor cells.

Most traditional studies on NS action mechanism suggest that NS could combine with p53 to inhibit its tumor suppressor function, and then lead to tumorigenesis (1). In addition, NS could protect telomere, its overexpression could regulate the telomere length negatively and then delay cell senescence; however, the absence of NS could increase the probability of telomere damage and mutation and then result in cell senescence (8). This may also be one type of mechanism of NS in tumorigenesis and tumor development.

However, in our previous studies, we used human acute myeloid leukemia cell line HL-60 which was p53-null as the research material. After inhibiting NS expression, HL-60 cells presented more apoptosis (9). Thus, we speculated that NS may also play its role independent of p53. Similar views have been reported by others, including Beekman et al (10), Jafamejad et al (11) and Nikpour et al (12). However, to date, only few studies have focused on the detailed mechanism of this pathway.

Clinically, the therapeutic effect of tumors is closely associated with p53. P53-null or mutate is one of the main reasons for drug-resistant, poor prognostic and therapeutic effects. Thus, exploring effective treatment targets and measures for p53-null and p53-mutate tumors is a critical issue that requires immediate attention. The present study analyzed the gene expression profiling changes of HL-60 cells after knocking down NS, aiming to explore the detailed mechanism of NS p53-independent pathway. These findings may lay the foundations for drug development for p53-null leukemia and even p53-null tumors.

\section{Materials and methods}

Cell culture. HL-60 cells were cultured in RPMI-1640 medium (Gibco-BRL) supplemented with $10 \%$ fetal bovine 
Table I. Sequences of two single-stranded DNA oligonucleotides.

\begin{tabular}{lccccc}
\hline No. & $5^{\prime}$ & STEM & Loop & STEM & $3^{\prime}$ \\
\hline NS-RNAi-1 & ccgg & agCAAGTATTGAAGTAGTAAA & CTCGAG & TTTACTACTTCAATACTTGCT & TTTTTg \\
NS-RNAi-2 & aattcaaaaa & agCAAGTATTGAAGTAGTAAA & CTCGAG & TTTACTACTTCAATACTTGCT & \\
\hline
\end{tabular}

serum (FBS; Gibco-BRL), $100 \mathrm{U} / \mathrm{ml}$ of penicillin, $100 \mu \mathrm{g} / \mathrm{ml}$ streptomycin and were incubated at $37^{\circ} \mathrm{C}$ with $5 \%$ humidified $\mathrm{CO}_{2}$. The cells were changed medium every 2 days.

Construction of NS-siRNA lentiviral vector. RNA interference (RNAi) sequence was designed corresponding to NS gene (Genbank NM_014366). Two single-stranded DNA oligonucleotides containing NS-RNAi sequence, loop circle, AgeI/EcoRI enzyme cutting site and termination signal sequence were synthesized (Table I) and annealed to a double-stranded DNA template. Subsequently, the template was inserted into the AgeI and EcoRI enzyme sites of GV248 lentiviral vector (Genechem, Shanghai, China) with catalysis by T4 DNA ligase to construct recombinant vectors expressing NS-shRNA. The recombinant vectors were transformed into competent E.coli and then confirmed by DNA sequencing. The recombinant vector and packaging vectors were co-transfected into $293 \mathrm{~T}$ cells, and the supernatants containing packaged vectors were harvested $48 \mathrm{~h}$ later. Subsequent purification using ultracentrifugation was performed and the titer of lentiviruses was determined.

Validation of the RNA-interference effect. Fresh medium for HL-60 cells was changed $24 \mathrm{~h}$ before transfection to ensure the cells in logarithmic phase at transfection. At the time of transfection, the cells were harvested and centrifuged, and then suspended in fresh medium with $10 \%$ FBS and seeded into a 6-well plate. A certain amount of NS-shRNA lentivirus was added to HL-60 cells according to lentiviral titer and MOI of HL-60 cells. At the same time, to exclude the influence of blank lentiviral vector and off-target silencing effect mediated by specific-shRNA, the blank control and the negative control group were also set. The same amount of blank lentiviral vectors and negative control lentivirus was added to HL-60 cells as the blank control group and negative control group, respectively. The final volume in each well was $2 \mathrm{ml}$. Sixteen hours after transfection, the medium was changed and continued to culture. Seventy-two hours after transfection, the plate was placed under inverted fluorescence microscope to observe the transfection efficiency.

Cells $\left(5-10 \times 10^{6}\right)$ in each group were harvested $96 \mathrm{~h}$ after transfection. Total-RNA was extracted from cells using TRIzol reagent (Invitrogen). The reverse transcription reaction was performed with PrimeScript ${ }^{\circledR}$ RT reagent Kit with gDNA Eraser (Takara). Expression of NS mRNA was detected by ABI 7500 real-time PCR instrument using SYBR ${ }^{\circledR}$ Premix Ex TapTMII (Tli RNaseH Plus) Kit (Takara). The sequence of primers for NS and GAPDH are shown in Table II. Relative gene expression was quantified to calculate the inhibition efficiency.
Table II. Primer pairs used for quantitative RT-PCR.

Gene $\quad$ Primer pairs

PIK3CD F: 5'-TTT CTC ATG GCT GTC CTT CAG-3

R: 5'-CAG GAG AAT CTA ACG GAT GC-3'

AKT2 F: 5'-CAT CAC ATC TGG TTT CCT TGG-3'

R: 5'-AAC TGG AAA TGT AAT TTT GGG-3'

STAT3 F: 5'-ACC AGC AAT ATA GCC GAT TCC-3'

R: 5'-CCA TTG GCT TCT CAA GAT ACC-3'

STAT5A F: 5'-ATT ATC TCA GCC CTG GTG ACC-3'

R: 5'-CTG CTG CTC ACT GAT GAT GGT-3'

GRB2 F: 5'-GGA CAT CCT CAA GGT TTT GAA C-3' R: 5'-CGC TCT CAC TCT CTC GGA TAA G-3'

HRAS F: 5'-AGC TGA TCC AGA ACC ATT TTG T-3' R: 5'-GTT GAT GGC AAA CAC ACA CAG-3'

MAPK9 F: 5'-CTG CGT CAC CCA TAC ATC AC-3' R: 5'-CTT TCT TCC AAC TGG GCA TC-3'

MAPK13 F: 5'-AGG TCT CTG GGG GTT GAG TTG GG-3' R: 5'-AGG GGC AGC AAC GTC TCA TTG C-3'

GAPDH F: 5'-TGA CTT CAA CAG CGA CAC CCA-3' R: 5'-CAC CCT GTT GCT GTA GCC AAA-3'

NS

F: 5'-TAGAGGTGTTGGATGCCAGAG-3'

R: 5'-CACGCTTGGTTATCTTCCCTTTA-3'

Microarray hybridization. Total RNA was purified using the RNase Mini Kit (Qiagen $\mathrm{p} / \mathrm{n}$ 74104), according to the protocol for Quick Amp Labeling Kit, One-Color (Agilent p/n 51900442), reverse transcript total-RNA to cDNA, and then further transcripted cDNA to cRNA with $\mathrm{Cy} 3$ labeling and purifying it. Next, the purified Cy3-labeled cRNA was used for hybridization on Agilent 4x44K Human Whole-Genome 60-mer oligonucleotide microarrays following the protocol for Agilent Gene Expression Hybridization Kit (p/n 5188-5242).

Microarray data acquisition, processing and analysis. Microarrays were scanned with Agilent DNA Microarray Scanner (Agilent p/n G2565BA), using the setting parameters recommended by Agilent Technologies (Green PMT were set at XDR Hi $100 \%$ and XDR Lo $10 \%$; scan resolution was set to $5 \mu \mathrm{m})$. Then, the Agilent Feature Extraction software v11.0.1.1 was used to analyze acquired microarray images, and the resulting text files extracted from it were imported into the GeneSpring GX v12.0 software package (Agilent 
A

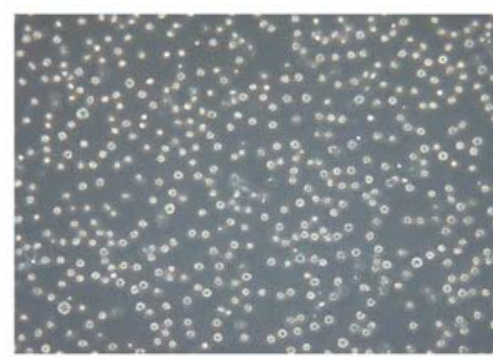

200X

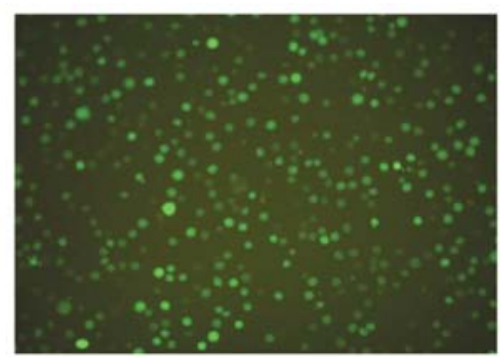

200X

B

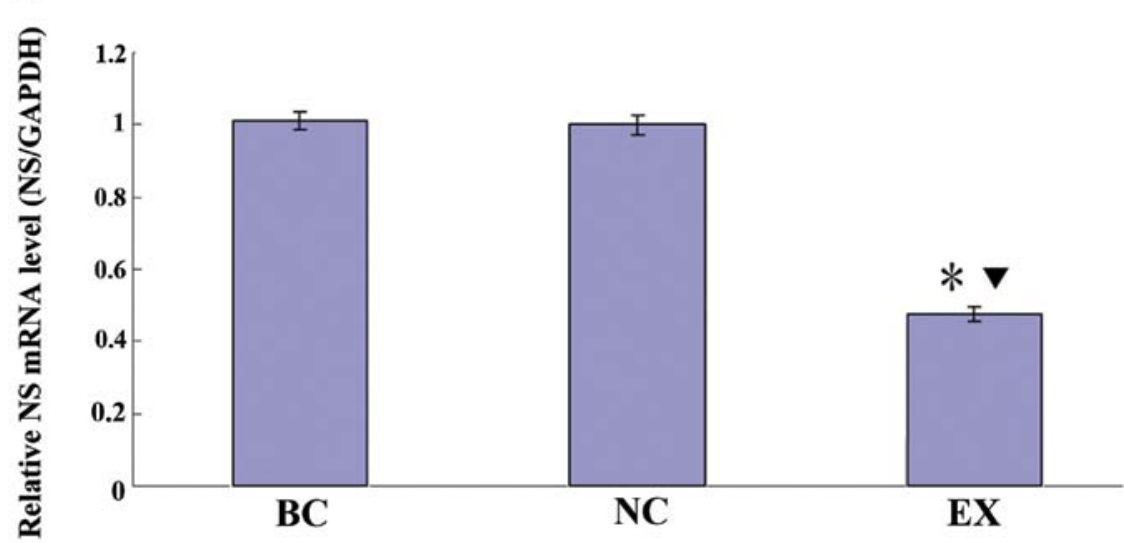

Figure 1. The interference effect of NS-shRNA lentivirus in HL-60 cells. (A) HL-60 cells transfected with NS-shRNA lentivirus were observed under an inverted microscope using general light (left) and fluorescence (right), respectively. (B) Detection of relative NS mRNA level in each group by real-time PCR. ${ }^{*} \mathrm{P}<0.05$ compared with the blank control (BC) group, ${ }^{\mathrm{P}} \mathrm{P}<0.05$ compared with the negative control (NC) group; EX, experimental group.

Technologies) for further analysis. After quantile normalization of the raw data, genes that at least $2 / 2$ samples have flags in Detected ('All Targets Value') were chosen for further data analysis. Differentially expressed genes with statistical significance were identified through fold-change filtering for 2 compared samples. Pathway analysis of the differentially expressed genes was performed using the KEGG Pathway Database (http://www.genome.jp/kegg).

Real-time quantitative PCR. Reverse transcription was performed using PrimeScript ${ }^{\circledR}$ RT reagent Kit with gDNA Eraser (Takara). Quantification of gene expression was performed by ABI 7500 real-time PCR instrument using SYBR ${ }^{\circledR}$ Premix Ex TapTMII (Tli RnaseH Plus) Kit (Takara). The expression level of GAPDH was used as an internal control. The expression of the following genes was analyzed: PIK3CD, AKT2, STAT3, STAT5A, GRB2, HRAS, MAPK9, MAPK13. The primers are listed in Table II.

Statistical analysis. The experiments were repeated at least three times and the data are presented as means \pm SD (standard deviation). Statistical software SPSS 17.0 was used for the assessment. The Student's t-test was used to compare means of two groups. Fisher's exact test was used to analyze the significance of the pathway. $\mathrm{P}<0.05$ was considered to indicate statistically significant differences.

\section{Results}

Downregulation of NS in HL-60 cells after NS-shRNA lentivirus transfection. The lentiviral vectors transfected HL-60 cells successfully and the transfection efficiency was $>80 \%$ which could be observed under an inverted fluorescence microscope (Fig. 1A). The NS mRNA expression was determined by real-time qPCR. As shown in Fig. 1B, there was no significant difference between the blank control group (BC) and the negative control group (NC; $\mathrm{P}>0.05)$; compared with the $\mathrm{BC}$ and the NC group, the levels of NS mRNA in the experimental group (EX) were significantly reduced by 52.9 and $52.3 \%$, respectively $(\mathrm{P}<0.05)$. To maximize reducing the off-target effect, we chose the NC and the EX group for further DNA microarray analysis.

Data analysis: general features. Total-RNA isolated from the EX and NC group cells was used to synthesize cDNA. Probe labeling, hybridization and scanning were performed by Shanghai Kangcheng Co., Ltd (China). To identify differentially expressed genes with statistical significance, a fold change filtering between two samples was performed and the default threshold was $\geq 2.0$ fold-change. Results showed that 2,628 genes, 818 upregulated and 1,810 downregulated, were differentially expressed in HL-60 cells following downregulation of NS.

Real-time $q P C R$ validation. To evaluate the reliability of the array results, eight differentially expressed genes (PIK3CD, AKT2, STAT3, STAT5A, GRB2, HRAS, MAPK9, MAPK13), which had >2-fold-changes according to the microarray assay results, were selected for further validation by real-time qPCR. The real-time qPCR results of these genes were generally in agreement with microarray data (Fig. 2). Although the fold-changes of these genes determined respec- 
Table III. Pathway analysis of upregulated genes.

\begin{tabular}{|c|c|c|c|}
\hline PathwayID & Definition & Fisher's P-value & Genes \\
\hline hsa05322 & $\begin{array}{l}\text { Systemic lupus } \\
\text { erythematosus - } \\
\text { Homo sapiens (human) }\end{array}$ & 7.77667E-06 & $\begin{array}{l}\text { C7//CTSG//ELANE//HIST1H2AC//HIST1H2AI//HIST1H2BB// } \\
\text { HIST1H2BF//HIST1H2BH//HIST1H2BK//HIST1H2BL// } \\
\text { HIST1H2BO//HIST1H3B//HIST1H3H//HIST1H4H//HIST1H4L// } \\
\text { HIST2H2BE//HIST2H2BF }\end{array}$ \\
\hline hsa05144 & $\begin{array}{l}\text { Malaria - } \\
\text { Homo sapiens (human) }\end{array}$ & 0.000416632 & CCL2//DARC//HBB//HBD//HGF//IL1B//IL8//THBS1 \\
\hline hsa04621 & $\begin{array}{l}\text { NOD-like receptor } \\
\text { signaling pathway - } \\
\text { Homo sapiens (human) }\end{array}$ & 0.001132948 & CASP5//CCL2//CXCL1//IL1B//IL8//MAPK13//MAPK9//NLRP1 \\
\hline
\end{tabular}

$\begin{array}{ll}\text { hsa04340 } & \text { Hedgehog } \\ & \text { signaling pathway - } \\ & \text { Homo sapiens (human) }\end{array}$

hsa05146 Amoebiasis -

Homo sapiens (human)

$\begin{array}{ll}\text { hsa04010 } & \text { MAPK } \\ & \text { signaling pathway - }\end{array}$

Homo sapiens (human)

hsa05020 Prion diseases -

Homo sapiens (human)

hsa05120 Epithelial cell signaling in Helicobacter pylori infection -

Homo sapiens (human)

hsa04620 Toll-like receptor signaling pathway -

Homo sapiens (human)

hsa04512 ECM-receptor interaction -

Homo sapiens (human)

hsa05132 Salmonella infection -

Homo sapiens (human)

hsa05217 Basal cell

carcinoma -

Homo sapiens (human)

hsa05200 Pathways in cancer -

Homo sapiens (human)

hsa04310 Wnt signaling pathway Homo sapiens (human)

0.01989438

hsa04962 Vasopressin-regulated water reabsorption -

Homo sapiens (human)

hsa04510 Focal adhesion -

Homo sapiens (human)

hsa04210

Apoptosis -

Homo sapiens (human)

hsa04722 Neurotrophin signaling pathway -

Homo sapiens (human)

0.003689235

0.004635966

0.007661671

0.007782237

0.01072619

0.01085929

0.01106789

0.01264349

0.01355671

0.01360474

0.02006348

0.02786726

0.03465665

0.03905394
CCND1//CYCS//FGF4//FGF8//FGFR3//FZD4//FZD5//FZD7// HGF//IL8//ITGAV//JUN//LAMC1//MAPK9//PTCH1//RAC1// RARA//RUNX1//SUFU//WNT4

CCND1//CSNK1E//FZD4//FZD5//FZD7//JUN//MAPK9//

PPP2R5B//PRKACB//RAC1//WNT4

COL11A2//CTSG//CXCL1//HSPB1//IL1B//IL8//LAMC1//

PRKACB//RAB5A//SERPINB13

CACNA1B//CACNA1E//FGF4//FGF8//FGFR3//FLNA//HSPB1// IL1B//JUN//MAPK13//MAPK8IP2//MAPK9//NTRK2// PLA2G2A//PRKACB//RAC1//RAPGEF2//RASGRP3

C7//EGR1//IL1B//LAMC1//PRKACB

CXCL1//IL8//JAM2//JUN//MAPK13//MAPK9//RAC1

CD44//COL11A2//ITGAV//LAMC1//SPP1//SV2B//SV2C// THBS 1

CXCL1//FLNA//IL1B//IL8//JUN//MAPK13//MAPK9//RAC1

FZD4//FZD5//FZD7//PTCH1//SUFU//WNT4

ARHGDIB//DYNLL2//PRKACB//RAB11A//RAB5A

CAPN2//CCND1//COL11A2//FLNA//HGF//ITGAV//JUN//

LAMC1//MAPK9//PXN//RAC1//SPP1//THBS1

ATM//CAPN2//CYCS//IL1B//IL1RAP//PRKACB//TNFRSF10C

ARHGDIB//JUN//MAPK13//MAPK9//NTRK2//PDK1//RAC1//

SH2B2//YWHAE 
Table III. Continued.

\begin{tabular}{llll}
\hline PathwayID & \multicolumn{1}{c}{ Definition } & Fisher's P-value & Genes \\
\hline hsa05014 & $\begin{array}{l}\text { Amyotrophic } \\
\text { lateral sclerosis (ALS) } \\
\text { Homo sapiens (human) }\end{array}$ & 0.04095384 & ALS2//CYCS//MAPK13//RAB5A//RAC1 \\
hsa04964 & $\begin{array}{l}\text { Proximal tubule } \\
\text { bicarbonate reclamation - } \\
\text { Homo sapiens (human) } \\
\text { Pertussis - } \\
\text { homo sapiens (human) }\end{array}$ & 0.0480162 & ATP1B1//GLS//SLC38A3 \\
& 0.0495752 & IL1B//IL8//JUN//LY96//MAPK13//MAPK9 \\
\hline
\end{tabular}

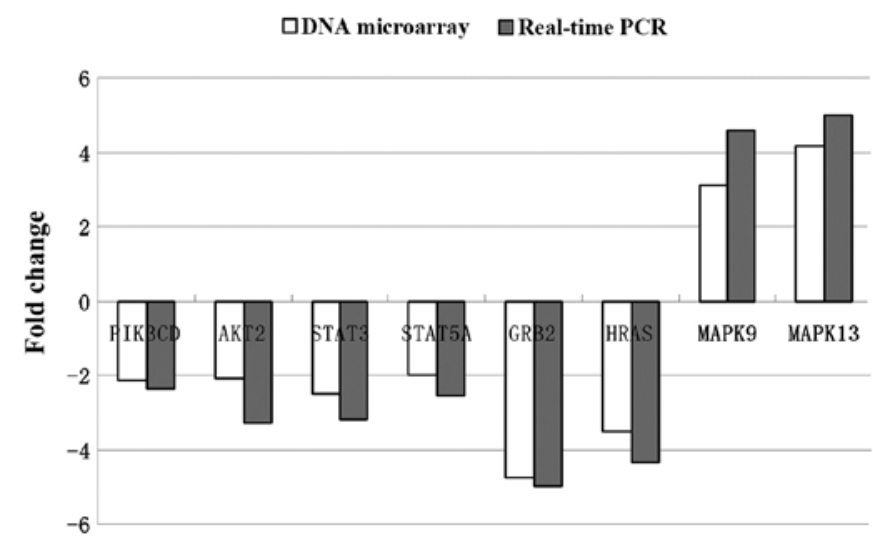

Figure 2. Quantitative real-time PCR confirmation of the microarray data. The real-time PCR results of these genes were in agreement with the microarray data. GAPDH was used as the internal control, and each sample was detected in triplicate.

tively by microarray and real-time qPCR were not completely the same, the general trends were. This was due to some noise that was inevitable. Nevertheless, these results still confirm our findings of differential gene expression by microarray analysis.

Pathway analysis. We proceeded with pathway analysis based on the KEGG database to illustrate all the available pathways containing differentially expressed genes. The significant pathways affected due to upregulated and downregulated genes are shown in Tables III and IV, respectively.

\section{Discussion}

Nucleostemin (NS) is a protein mainly expressed on stem and tumor cells, and it is important to maintain early embryonic development and tumor cell proliferation $(1,3,4)$. Most studies consider that NS could inhibit the antineoplastic function of p53 through combining with it (1), but some researchers still consider that NS could also play its function independent of p53 (10-15). However, the specific mechanism of this p53-independent pathway has yet to be elucidated.

In this study, we used lentivirus-mediated RNA interference and DNA microarray technology to investigate the differences of gene expressing profiling after knocking down NS in HL-60 cells, which was p53-null, in order to further explore the specific mechanism of NS p53-independent pathway. Our study showed that knockdown of NS in HL-60 cells could modulate the expression of extensive genes. Next, we proceeded to pathway analysis to explore how NS plays its function independent of p53. In all the pathways which changed significantly, the acute myeloid leukemia pathway (Pathway ID: has05221) warranted further attention, since HL-60 was an acute myeloid leukemia cell line. The PI3K-AKT, JAK-STAT and MAPK pathways are three main pathways included in acute myeloid leukemia (Fig. 3).

The PI3K-AKT pathway is one of the most important cellular signal transduction pathways involved in the regulation of proliferation. It plays its role of inhibiting apoptosis and promoting proliferation in cells through impacting the activated state of a variety of downstream effectors, and is closely related to the occurrence of many human tumors. After PI3K is activated by upstream molecules, it phosphorylates PI $(4,5)$ $\mathrm{P} 2$ on plasma membrane to PI $(3,4,5) \mathrm{P} 3$, the latter could translocate AKT to plasma membrane to activate it. Next, the activated AKT could activate or inhibit its downstream target proteins such as Bad, Caspase 9, NF- $\kappa$ B, Forkhead, mTOR, Par-4 and P21 through phosphorylation, which mediate cell growth and promote cell survival induced by insulin and types of growth factors; it is an important antiapoptotic factor $(16,17)$. In addition, AKT could regulate the activity of IKK, cause the nuclear translocation of $N F-\kappa B$, and then promote the transcription of $\mathrm{NF}-\kappa \mathrm{B}$-dependent survival genes to facilitate cell survival (18); also, the inhibition of $\mathrm{NF}-\kappa \mathrm{B}$ could promote cell apoptosis (19). In this research, after knocking down NS expression in HL-60 cells, the key point in the PI3K-AKT pathway including PI3K (PIK3CD), AKT2, IKK (IкBKG) and $\mathrm{NF}-\kappa \mathrm{B}$ were all downregulated (Fig. 3). This suggested that the inhibition of the PI3K/AKT/NF- $\mathrm{B}$ pathway may participate in the apoptosis caused by knocking down NS in HL-60 cells.

The continuous activation of the JAK-STAT pathway is widespread in leukemia cells; the activated key point STAT could induce abnormal expression of genes closely related to cell proliferation, differentiation and apoptosis, and then promote cell proliferation and inhibit apoptosis through various pathways $(20,21)$. STAT family members are closely related to tumors, especially STAT3 and STAT5A. Some studies reported that inhibition of STAT5A could block the 
Table IV. Pathway analysis of downregulated genes.

\begin{tabular}{llll}
\hline PathwayID & \multicolumn{1}{c}{ Definition } & Fisher's P-value & \multicolumn{1}{c}{ Genes } \\
\hline hsa03040 & Spliceosome - & \multirow{2}{*}{ 3.71589E-08 } & ACIN1//BUD31//CHERP//CTNNBL1//DDX39B//DHX38// \\
& & HNRNPA1L2//HNRNPA3//HNRNPC//HNRNPK//HSPA8// \\
& & ISY1//LSM4//NHP2L1//PPIE//PQBP1//PRPF31//PRPF6// \\
& & PRPF8//RBMX//SF3B2//SF3B4//SNRNP40//SNRNP70// \\
& & SNRPA//SNRPB//SNRPC//SRSF3//SRSF9//TCERG1//THOC1// \\
& & THOC3//THOC4//TRA2B
\end{tabular}

hsa03013 RNA transport -

Homo sapiens (human)

hsa03410 Base excision repair -

Homo sapiens (human)

hsa00520 Amino sugar and nucleotide sugar metabolism -

Homo sapiens (human)

hsa05221 Acute myeloid leukemia Homo sapiens (human)

hsa04142 Lysosome -

Homo sapiens (human)

hsa04141 Protein processing in endoplasmic reticulum Homo sapiens (human)

\section{hsa03050 Proteasome - \\ Homo sapiens (human) \\ hsa05213 Endometrial cancer - Homo sapiens (human) \\ hsa04666 Fc $\gamma$ R-mediated phagocytosis - Homo sapiens (human)}

hsa04662 B cell receptor signaling pathway Homo sapiens (human)

hsa00310 Lysine degradation Homo sapiens (human)

hsa04910 Insulin signaling pathway Homo sapiens (human)

hsa05130 Pathogenic

Escherichia coli infection Homo sapiens (human)

hsa04150 mTOR signaling pathway Homo sapiens (human)
$2.51665 \mathrm{E}-05$

AAAS//ACIN1//DDX39B//EEF1A1//EIF2B4//EIF2B5//EIF3C// EIF3F//EIF3G//EIF4A1//EIF4B//EIF4G1//GEMIN8//NUP188// NUP210//NUP214//NUP62//NUP93//NXF1//POM121// PRMT5//RNPS1//SEC13//SNUPN//TACC3//THOC1//THOC3// THOC4//THOC5//THOC6//WIBG//XPO5

0.000263071 APEX1//FEN1//HMGB1//LIG1//MPG//NTHL1//OGG1// PARP1//POLD2//POLE//XRCC1

0.00053131 GALE//GALK1//GALT//GMPPA//GMPPB//GNPDA1// GNPNAT1//HEXA//NAGK//NPL//PMM1//PMM2//TSTA3

0.001151056

AKT2//ARAF//CEBPA//GRB2//HRAS//IKBKG//MAP2K2// PIK3CD//RAF1//RELA//RPS6KB1//STAT3//STAT5A//ZBTB16

0.001392349

ABCA2//AP1M1//AP4B1//ATP6AP1//ATP6V0D1//CLTCL1//

CTSA//CTSC//CTSD//CTSL1//DNASE2//GALNS//GBA//

GGA1//GNPTG//HEXA//IGF2R//LAMP2//MAN2B1//

MCOLN1//NAGLU//PPT1//PSAP

0.001583112 AMFR//BAK1//CANX//CAPN1//DDIT3//DNAJC5G//EDEM2// ERP29//HSP90AA1//HSP90AB1//HSPA8//LMAN1L//LMAN2// MAN1B1//NSFL1C//P4HB//PDIA4//PPP1R15A//PRKCSH// RAD23B//RPN1//RRBP1//SEC13//SEC61A1//SSR2//STUB1// UBE2J2//UBQLN4//UBXN6

0.003429877 PSMA7//PSMB9//PSMC3//PSMC4//PSMC5//PSMD13// PSMD2//PSMD3//PSMD8//PSME1//PSMF1

0.003806897 AKT2//ARAF//AXIN1//ELK1//FOXO3//GRB2//HRAS//ILK// MAP2K2//PDPK1//PIK3CD//RAF1

0.004610906 AKT2//ARPC1B//CFL1//DNM2//GSN//HCK//LAT//NCF1// PIK3CD//PIP5K1C//PRKCD//PTPRC//RAC2//RAF1// RPS6KB1//VASP//WAS//WASF2

0.005562133 AKT2//CD79B//CD81//GRB2//HRAS//IFITM1//IKBKG// LILRB3//MAP2K2//NFKBIB//PIK3CD//PTPN6//RAC2// RAF1//RELA

0.006858597 DOT1L//EHMT2//GCDH//GLT25D1//HADHA//MLL4// PLOD1//SETD1B//SETD2//SUV39H1//WHSC1

0.007757841 AKT2//ARAF//CALML3//ELK1//EXOC7//FLOT1//FLOT2// GRB2//HRAS//MAP2K2//MKNK2//PCK2//PDPK1//PHKG2// PIK3CD//PPP1CA//PRKAG1//PRKAR1B//PYGB//RAF1// RPS6//RPS6KB1//TSC2

0.008284432 ABL1//ARHGEF2//ARPC1B//TUBA1A//TUBA1C//TUBA3C// TUBA4A//TUBB//TUBB2C//TUBB8//WAS//YWHAZ

0.0108534 
Table IV. Continued.

\begin{tabular}{|c|c|c|c|}
\hline PathwayID & Definition & Fisher's P-value & Genes \\
\hline hsa04722 & $\begin{array}{l}\text { Neurotrophin } \\
\text { signaling pathway - } \\
\text { Homo sapiens (human) }\end{array}$ & 0.01165631 & $\begin{array}{l}\text { ABL1//AKT2//ARHGDIA//CALML3//CAMK2G//FOXO3// } \\
\text { GRB2//HRAS//IRAK1//MAP2K2//MAPKAPK2//NFKBIB// } \\
\text { PIK3CD//PRKCD//RAF1//RELA//RPS6KA1//RPS6KA4// } \\
\text { YWHAE//YWHAZ//ZNF274 }\end{array}$ \\
\hline hsa00511 & $\begin{array}{l}\text { Other glycan degradation - } \\
\text { Homo sapiens (human) }\end{array}$ & 0.02057153 & GBA//HEXA//MAN2B1//MAN2B2//MAN2C1 \\
\hline hsa03030 & $\begin{array}{l}\text { DNA replication - } \\
\text { Homo sapiens (human) }\end{array}$ & 0.0211445 & FEN1//LIG1//MCM5//MCM7//POLA2//POLD2//POLE//RFC2 \\
\hline hsa00480 & $\begin{array}{l}\text { Glutathione metabolism - } \\
\text { Homo sapiens (human) }\end{array}$ & 0.02164977 & $\begin{array}{l}\text { G6PD//GGT1//GPX2//GPX4//GSS//GSTK1//GSTZ1//IDH2// } \\
\text { PGD//SRM }\end{array}$ \\
\hline hsa05140 & $\begin{array}{l}\text { Leishmaniasis - } \\
\text { Homo sapiens (human) }\end{array}$ & 0.02422244 & $\begin{array}{l}\text { C3//CYBA//ELK1//IRAK1//ITGA4//ITGB2//NCF1//NCF4// } \\
\text { NFKBIB//PTPN6//RELA//TAB1//TGFB1 }\end{array}$ \\
\hline hsa05220 & $\begin{array}{l}\text { Chronic myeloid leukemia - } \\
\text { Homo sapiens (human) }\end{array}$ & 0.02422244 & $\begin{array}{l}\text { ABL1//AKT2//ARAF//CTBP1//GRB2//HRAS//IKBKG// } \\
\text { MAP2K2//PIK3CD//RAF1//RELA//STAT5A//TGFB1 }\end{array}$ \\
\hline hsa03015 & $\begin{array}{l}\text { mRNA } \\
\text { surveillance pathway - } \\
\text { Homo sapiens (human) }\end{array}$ & 0.03338131 & $\begin{array}{l}\text { ACIN1//CPSF1//DDX39B//NXF1//PABPN1//PPP2R1A// } \\
\text { PPP2R3B//PPP2R5D//RNGTT//RNPS1//SMG5//SMG6// } \\
\text { THOC4//WIBG }\end{array}$ \\
\hline hsa03420 & $\begin{array}{l}\text { Nucleotide excision repair - } \\
\text { Homo sapiens (human) }\end{array}$ & 0.04168682 & $\begin{array}{l}\mathrm{DDB} 2 / / \mathrm{ERCC} 1 / / \mathrm{ERCC} 2 / / \mathrm{GTF} 2 \mathrm{H} 4 / / \mathrm{LIG} 1 / / \mathrm{POLD} 2 / / \mathrm{POLE} / / \\
\mathrm{RAD} 23 \mathrm{~B} / / \mathrm{RFC} 2\end{array}$ \\
\hline
\end{tabular}

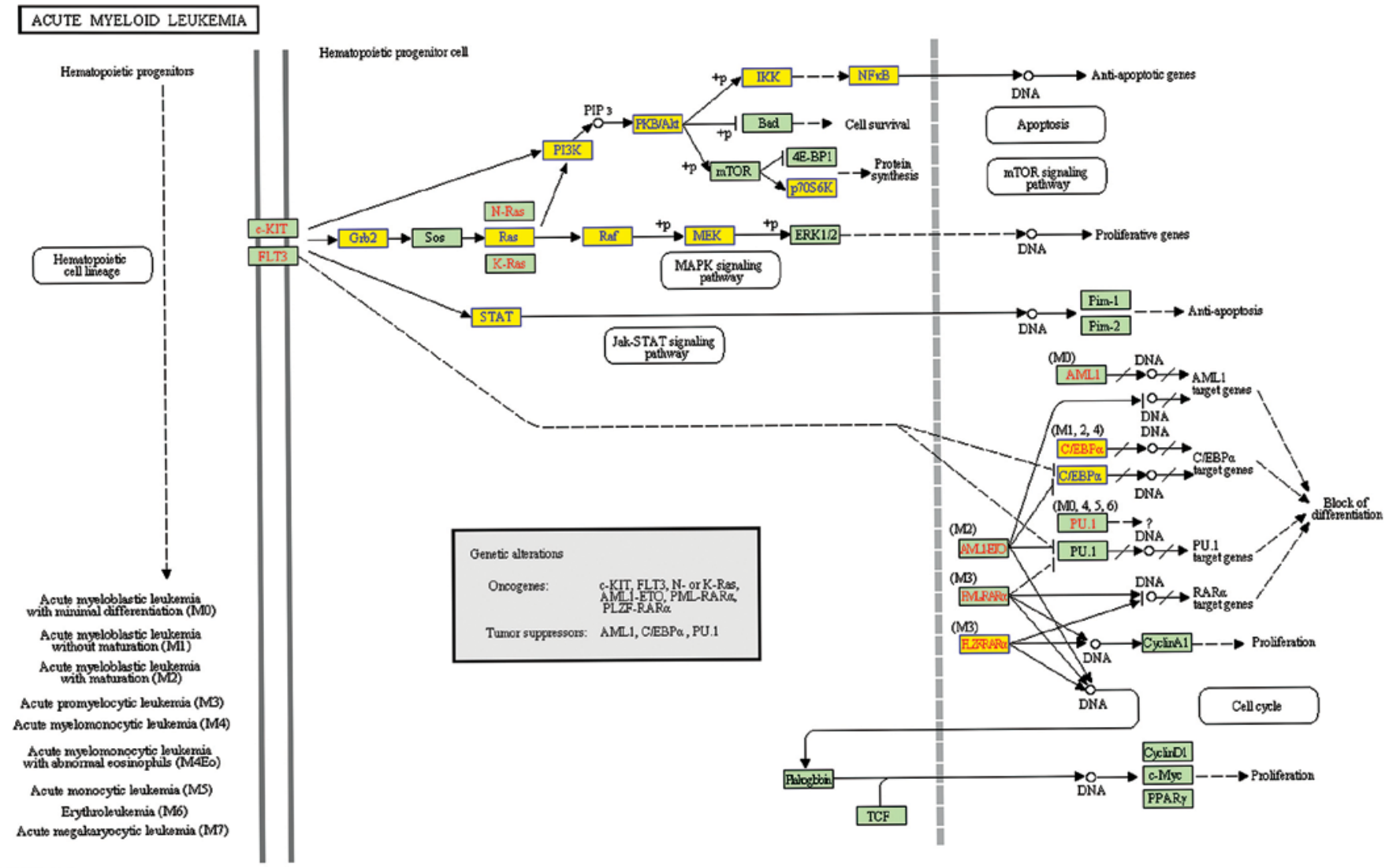




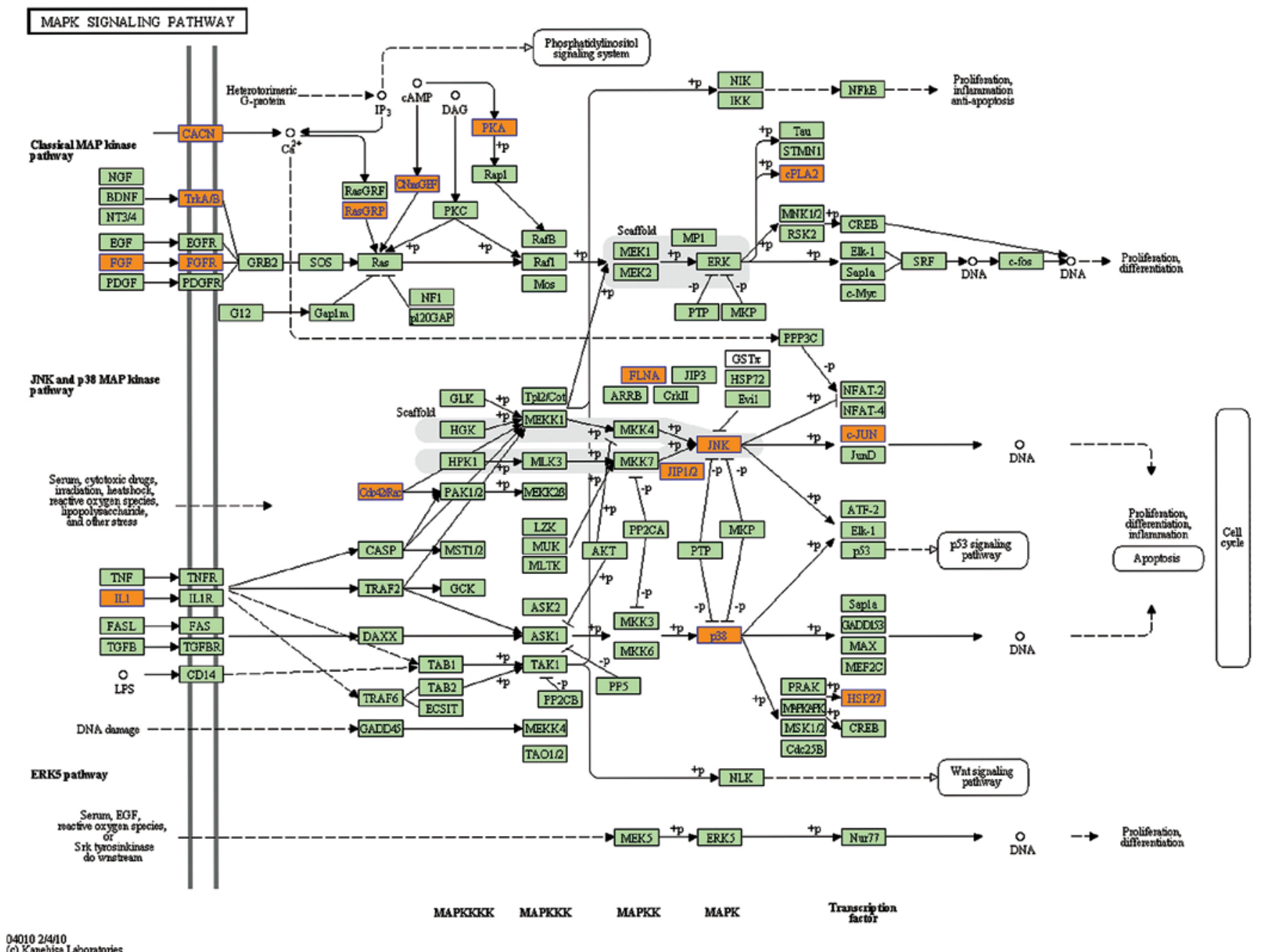

Figure 4. MAPK signaling pathway. Orange marked nodes are associated with upregulated or only whole dataset genes, green nodes have no significance.

growth of prostate cancer cells (22); inhibition of STAT3 could promote the apoptosis of bladder cancer cells (23); high-dose methylprednisone induced HL-60 cell apoptosis, and this process was accompanied by downregulation of STAT5A protein (24). In the present study, STAT3 and STAT5A were both downregulated after the inhibition of NS, indicating that downregulation of STAT may be another reason for cell apoptosis after knocking down NS in HL-60 cells.

Mitogen-activated protein kinase (MAPK) is a type of serine-threonine protein kinase existing in cells and plays an important role in cell signal transduction. MAPK pathways mainly include the RAS-RAF-MEK-ERK1/2, the JNK and the P38MAPK pathway (25). A previous study reported that the RAS-RAF-MEK-ERK1/2 pathway may have an important impact on the proliferation and differentiation of melanoma cells (26); inhibiting this pathway could be an effective approach for antitumor therapy (27-29). Some antineoplastic drugs could induce cell apoptosis mainly through the activation of JNK and P38MAPK pathway (30-32). Herein, we found genes involved in the RAS-RAF-MEK-ERK1/2 pathway, including GRB2, RAS (HRAS), RAF (RAF1), MEK2 (MAP2K2), were all downregulated (Fig. 3), while JNK (MAPK9) and P38 (MAPK13) were upregulated (Fig. 4). This suggested that the inhibition of RAS-RAF-MEK-ERK1/2 pathway and activation of JNK as well as P38MAPK pathway may also involved in HL-60 cell apoptosis caused by knocking down the expression of NS.

The pathway analysis of downregulated genes showed that spliceosome (Pathway ID: hsa03040) and RNA transport (Pathway ID: hsa03013) were much more significant. Spliceosome plays a critical role in processing pre-mRNA and folding mRNA. The data of our research showed that many genes participated in the formation of spliceosome, therefore it was speculated that knocking down NS may influence the cellular gene expression through preventing RNA processing and transport. Romanova et al presented a similar viewpoint, that knocking down NS had an impact on the processing of rRNA, folding and stabilization of other RNAs $(33,34)$.

In conclusion, we detected the changes of gene expression profiles in HL-60 cells which were p53-null after knocking down NS expression, in order to explore how NS plays its function without the existence of p53. The DNA microarray data showed a large number of genes were differentially expressed. Pathway analysis indicated that after NS was inhibited in HL-60 cells, especially inhibition of the PI3K-AKT pathway, the JAK-STAT pathway and RAS-RAF-MEK-ERK1/2, and the activation of JNK pathway as well as P38MAPK pathway may be involved in the cell apoptosis caused by knocking down 
NS. This study provides insight into exploring the functional mechanism of NS independent of p53, and lays the foundations for the search of new effective therapeutic targets of p53-null leukemia and even p53-null tumors.

\section{Acknowledgements}

This study was supported by grants from the National Natural Science Foundation of China (No. 81271911) and the Key Projects of Medical Science and Technology of Henan Province (No. 201002006). The authors thank Professor Guoqiang Zhao, Basic Medical School of Zhengzhou University, for his valuable advice on transfection and RNA-interference technology. We also acknowledge the Kangcheng company in Shanghai, China, for DNA microarray technical help.

\section{References}

1. Tsai RY and McKay RD: A nucleolar mechanism controlling cell proliferation in stem cells and cancer cells. Genes Dev 16: 2991-3003, 2002.

2. Liu SJ, Cai ZW, Liu YJ, Dong MY, Sun LQ, Hu GF, et al: Role of nucleostemin in growth regulation of gastric cancer, liver cancer and other malignancies. World J Gastroenterol 10: 1246-1249, 2004.

3. Liu RL, Xu Y, Zhang ZH, Wang M, Sun JT, Qi SY, et al: Expression of nucleostemin in prostate cancer tissues and its clinical significance. Zhonghua Nan Ke Xue 14: 418-422, 2008 (In Chinese)

4. Zhang GY, Yin L, Li SL, Xing WY, Zhao QM, Le XP, et al: Expression of nucleostemin mRNA and protein in the esophageal squamous cell carcinoma. Zhonghua Zhong Liu Za Zhi 30 125-128, 2008 (In Chinese).

5. Sijin L, Ziwei C, Yajun L, Meiyu D, Hongwei Z, Guofa H, et al: The effect of knocking-down nucleostemin gene expression on the in vitro proliferation and in vivo tumorigenesis of HeLa cells. J Exp Clin Cancer Res 23: 529-538, 2004.

6. Ma H and Pederson T: Depletion of the nucleolar protein nucleostemin causes $\mathrm{G} 1$ cell cycle arrest via the 553 pathway. Mol Biol Cell 18: 2630-2635, 2007.

7. Liu RL, Zhang ZH, Zhao WM, Wang M, Qi SY, Li J, et al: Expression of nucleostemin in prostate cancer and its effect on the proliferation of PC-3 cells. Chin Med J (Engl) 121: 299-304, 2008.

8. Hsu JK, Lin T and Tsai RY: Nucleostemin prevents telomere damage by promoting PML-IV recruitment to SUMOylated TRF1. J Cell Biol 197: 613-624, 2012.

9. Yue BH, Yu LN and Wang YY: Effects of silent nucleostemin gene expression on apoptosis of HL-60 cells in vitro. Zhongguo Shi Yan Xue Ye Xue Za Zhi 17: 319-323, 2009 (In Chinese).

10. Beekman C, Nichane M, De Clercq S, Maetens M, Floss T, Wurst W, et al: Evolutionarily conserved role of nucleostemin: controlling proliferation of stem/progenitor cells during early vertebrate development. Mol Cell Biol 26: 9291-9301, 2006.

11. Jafamejad SM, Mowla SJ and Matin MM: Knocking-down the expression of nucleostemin significantly decreases rate of proliferation of rat bone marrow stromal stem cells in an apparently p53-independent manner. Cell Prolif 41: 28-35, 2008.

12. Nikpour P, Mowla SJ, Jafarnejad SM, Fischer U and Schulz WA: Differential effects of Nucleostemin suppression on cell cycle arrest and apoptosis in the bladder cancer cell lines 5637 and SW1710. Cell Prolif 42: 762-769, 2009.

13. Liu R, Zhang $\mathrm{Z}$ and $\mathrm{Xu} \mathrm{Y}$ : Downregulation of nucleostemin causes G1 cell cycle arrest via a p53-independent pathway in prostate cancer PC-3 cells. Urol Int 85: 221-227, 2010.

14. Zwolinska AK, Heagle WA, Beekman C, Sedivy JM and Marine JC: Suppression of Myc oncogenic activity by nucleostemin haploinsufficiency. Oncogene 31: 3311-3321, 2012.
15. Paridaen JT, Janson E, Utami KH, Pereboom TC, Essers PB, van Rooijen C, et al: The nucleolar GTP-binding proteins Gnl2 and nucleostemin are required for retinal neurogenesis in developing zebrafish. Dev Biol 355: 286-301, 2011.

16. Osaki M, Oshimura M and Ito H: PI3K-Akt pathway: its functions and alterations in human cancer. Apoptosis 9: 667-676, 2004.

17. Song G, Ouyang G and Bao S: The activation of Akt/PKB signaling pathway and cell survival. J Cell Mol Med 9: 59-71, 2005.

18. Vandermoere F, El Yazidi-Belkoura I, Adriaenssens E, Lemoine J and Hondermarck $\mathrm{H}$ : The antiapoptotic effect of fibroblast growth factor-2 is mediated through nuclear factor-kappaB activation induced via interaction between Akt and IkappaB kinase-beta in breast cancer cells. Oncogene 24: 5482-5491, 2005.

19. Zanotto-Filho A, Delgado-Cañedo A, Schröder R, Becker M, Klamt F and Moreira JC: The pharmacological NFkappaB inhibitors BAY117082 and MG132 induce cell arrest and apoptosis in leukemia cells through ROS-mitochondria pathway activation. Cancer Lett 288: 192-203, 2010.

20. Yu H and Jove R: The STATs of cancer - new molecular targets come of age. Nat Rev Cancer 4: 97-105.

21. Haura EB, Turkson J and Jove R: Mechanisms of disease: Insights into the emerging role of signal transducers and activators of transcription in cancer. Nat Clin Pract Oncol 2: 315-324, 2005.

22. Dagvadorj A, Kirken RA, Leiby B, Karras J and Nevalainen MT: Transcription factor signal transducer and activator of transcription 5 promotes growth of human prostate cancer cells in vivo. Clin Cancer Res 14: 1317-1324, 2008.

23. Chen CL, Cen L, Kohout J, Hutzen B, Chan C, Hsieh FC, et al: Signal transducer and activator of transcription 3 activation is associated with bladder cancer cell growth and survival. Mol Cancer 7: 78, 2008.

24. Kaymaz BT, Selvi N, Saydam G, Sahin F and Kosova B: Methylprednisolone induces apoptosis by interacting with the JAK/STAT pathway in HL-60 and K-562 leukemic cells. Hematology 17: 93-99, 2012.

25. Raman M, Chen W and Cobb MH: Differential regulation and properties of MAPKs. Oncogene 26: 3100-3112, 2007.

26. Busca R, Abbe P, Mantoux F, Aberdam E, Peyssonnaux C, Eychène $\mathrm{A}$, et al: Ras mediates the cAMP-dependent activation of extracellular signal-regulated kinases (ERKs) in melanocytes. EMBO J 19: 2900-2910, 2000.

27. Huang D, Ding Y, Luo WM, Bender S, Qian CN, Kort E, et al: Inhibition of MAPK kinase signaling pathways suppressed renal cell carcinoma growth and angiogenesis in vivo. Cancer Res 68: 81-88, 2008.

28. Ji Z, Flaherty KT and Tsao H: Targeting the RAS pathway in melanoma. Trends Mol Med 18: 27-35, 2012.

29. Daouti S, Wang H, Li WH, Higgins B, Kolinsky K, Packman K, et al: Characterization of a novel mitogen-activated protein kinase kinase $1 / 2$ inhibitor with a unique mechanism of action for cancer therapy. Cancer Res 69: 1924-1932, 2009.

30. Su JC, Lin KL, Chien CM, Lu CM, Chen YL, Chang LS and Lin SR: Novel indoloquinoline derivative, IQDMA, induces $\mathrm{G}(2) / \mathrm{M}$ phase arrest and apoptosis in A549 cells through JNK/p38 MAPK signaling activation. Life Sci 85: 505-516, 2009.

31. Brosseau CM, Pirianov G and Colston KW: Involvement of stress activated protein kinases (JNK and p38) in 1,25 dihydroxyvitamin D3-induced breast cell death. Steroids 75: 1082-1088, 2010.

32. Uehara N, Kanematsu S, Miki H, Yoshizawa K and Tsubura A: Requirement of p38 MAPK for a cell-death pathway triggered by vorinostat in MDA-MB-231 human breast cancer cells. Cancer Lett 315: 112-121, 2012.

33. Romanova L, Grand A, Zhang L, Rayner S, Katoku-Kikyo N, Kellner S, et al: Critical role of nucleostemin in pre-rRNA processing. J Biol Chem 284: 4968-4977, 2009.

34. Romanova L, Kellner S, Katoku-Kikyo N and Kikyo N: Novel role of nucleostemin in the maintenance of nucleolar architecture and integrity of small nucleolar ribonucleoproteins and the telomerase complex. J Biol Chem 284: 26685-26694, 2009. 